\title{
Research on the Relationship between Crop Cost and Price
}

\author{
Senhua Wang, Rui Li \\ School of Economics and Management, Beihang University, Beijing, 100191, China
}

\begin{abstract}
Based on the analysis on the grain cost and price, we analyze the main reason for the grain consumer price rising with statistical methods. The result illustrates that distinguishing the consumer price and production price plays an important role to research the relationship between them. Farmers' don't get any benefits from grain price rising, but afford most of the material factors cost. It is the circulation cost not the production cost that promotes the consumer price rising.
\end{abstract}

Keywords: Production Cost; Circulation Cost; Consumer Price; Production Price.

\section{粮食成本与粮食价格的关系研究}

\section{王森华，李锐}

北京航空航天大学 经济管理学院, 北京市, 中国

摘要: 在对粮食成本和粮食价格进一步细分的基础上, 运用统计分析方法对粮食消费价格上 涨的主要原因进行了深入分析。研究发现正确区分消费价格和生产价格以及正确区分生产价 格成本和消费价格成本, 对于研究成本和价格二者之间的关系具有重要的影响; 此外, 研究 得出的结论是：农民不仅仅没有从粮食价格上涨中获得重大利益而且承担了相当大部分的要 素成本上升; 推动最终消费价格的大幅上涨的原因是流通成本而不是生产成本。

关键词: 生产成本; 流通成本; 消费价格; 生产价格

\section{1. 前言}

粮食问题关系到国家大局的稳定，受到政府和社会的广泛关注，粮食价格更是关注的焦点。 近年来，诸多学者对于粮价上涨这一现象从供求关系、通货膨胀、成本要素以及资本炒作等 不同角度进行了深入研究, 但是对粮食价格和成本之间的关系的研究相对较少。目前相关研 究主要从以下两个方面展开：对粮食成本和价格的关系侧重于理论分析, 这一方面的研究是 从多种经济因素对农产品的价格上涨进行讨论，成本作为其中的一个因素进行讨论 [1]; 另一 方面是对粮食成本和价格的关系侧重于实证分析, 从生产成本、流通成本多个角度进行研究, 研究流通成本的相对较少 $[2,3]$ 。

这些都从成本角度对农产品价格进行了研究，但没有对粮食不同类型的成本和粮食的不同 价格进行具体的细分, 导致了某些结论不太符合实际情况。有学者提出应当在研究中区分不 同类型价格和不同类型成本的含义 [4], 研究的意义在于明确阐述不同的粮食成本与不同的粮 食价格之间的关系，并在此基础上对粮食价格上涨的原因和影响进行深入研究 [5]。

\section{2. 粮食作物的不同成本与价格的含义及关系框架}

\section{1. 样本数据}

小麦、玉米、水稻这三种最主要的粮食作物，无论种植面积还是产量都占了我国粮食作物

的 70\%以上。“三种粮食平均” 指稻谷、小麦、玉米产量的平均值, 采用的是不包括香港、 澳门特别行政区和台湾省在内的全国性数据。数据来源是历年的《全国农产品成本收益资料 汇编》与国家统计局网站上的相关数据。全国农产品成本收益资料来源于各级价格主管部门 成本调查队及烟草、供销等相关部门对全国 1553 个县 60000 多农户的典型调查汇总数据。在 
分析三种主要粮食作物平均物质成本的变化特点及影响时采用 1975-2014 年的绝对数据。在 分析粮食消费价格时由于没有粮食消费价格的绝对平均数据, 采用 1990-2014 年期间的相对 数据进行分析, 2000 年以前粮食生产价格总指数为粮食收购价格指数。

通过对粮食作物的不同成本与不同价格进行区分比较，分析不同成本之间、不同价格之间 以及不同成本与价格之间的关系框架 $[6,7]$ 。

\section{2. 粮食作物的价格和成本}

粮食价格分为粮食生产价格和粮食消费价格 [8]。粮食生产价格是指农业生产者首次出售 其生产的粮食时的价格。产品价格是由生产成本和平均利润决定的, 其中产品成本约占价格 的 70\%。因此, 农产品价格是依据产品成本这一因素, 以产品成本确定产品价格。粮食消费 价格是居民从超市或集贸市场购买粮食时所支付的价格。全国居民消费食品价格指数是按照 居民消费内容对全国居民消费价格指数的一个子类，反映居民家庭购买粮食项目费用价格变 动趋势和程度的相对数。粮食生产价格和粮食消费价格虽然都是粮食价格, 但是二者之间的 差别是很大的。粮食生产价格对应生产环节，包括粮食生产成本及对应的利润。粮食消费价 格对应流通环节，是在粮食流通成本上加上粮食流通过程中产生的各种成本和利润。

粮食作物的物质成本指在直接生产过程中消耗的各种农业生产资料的费用、购买各项服务 的支出以及与生产相关的其他实物或现金支出, 包括直接费用和间接费用两部分。直接费用 包括种子费、化肥费用、农膜费用、农药费用、蓄力费、机械作业费、排灌费、租赁作业费 以及其它直接费用; 间接费用包括固定资产折旧、税金、保险费、管理费、财务费以及销售 费用。粮食作物的生产成本包括直接费用、间接生产费用以及人工成本。粮食作物的总成本 指生产过程中耗费的现金、实物、劳动力和土地等所有资源的成本。三种主要粮食作物的总 成本在农业税取消之前对应的生产成本加上相应的农业税; 在农业税取消之后是对应的生产 成本加上对应的土地成本。土地成本, 也称地租, 指土地作为一种生产要素投入到生产中的 成本, 包括流转地租金和自营地折租。人工成本指生产过程中直接使用的劳动力成本, 包括 家庭用工折价和雇工费用两部分。家庭用工是指生产者和家庭成员的劳动、与他人相互换工 的劳动以及他人单方无偿提供的劳动用工。商品流通是指商品或服务从生产领域向消费领域 的转移过程。流通成本是流通主体为了完成产品由生产者向消费者转移进行的资源总投入, 表现为在运输、仓储、包装、加工、配送等不同流通阶段或在商流、物流、资金流、信息流 等不同流通内容上的费用支出总和。

2.3. 粮食作物成本与价格的关系框架

粮食各种成本之间的关系用结构图来表示（图 1)。粮食消费价格对应的是生产环节粮食 总成本和流通成本之和，粮食生产价格对应的是生产环节的总成本。

\section{3. 三种主要粮食作物的不同成本及价格的历史动态分析}

研究对象为粮食作物中的三种主要粮食作物: 稻谷、小麦、玉米, 针对三种作物的平均数 据进行的研究 (图 1)。

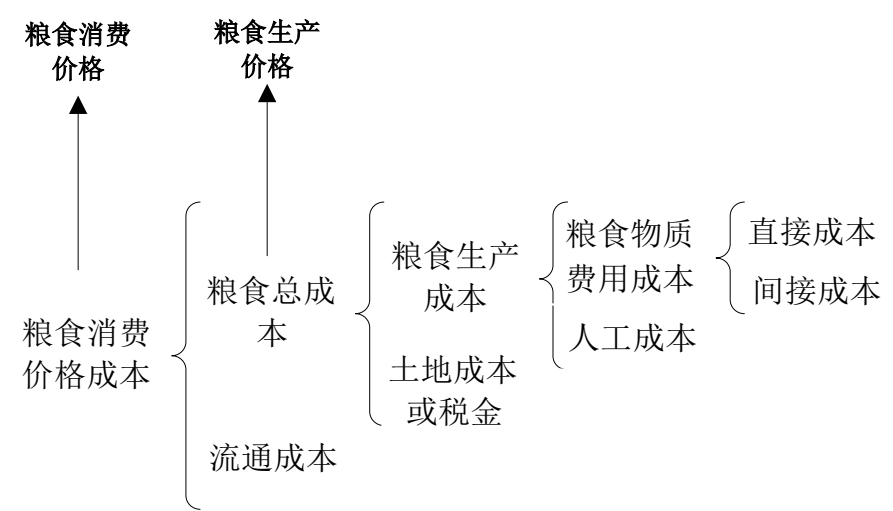

图1 粮食作物成本与价格的关系 


\section{1 三种主要粮食作物的生产总成本分析}

（1）三种主要粮食作物物质成本要素的构成变化及影响

三种主要粮食作物的生产成本中, 物质费用成本占比重最大。每亩生产成本中物质成本所 占比例在 40 年间的平均值约为 58\%。与此同时中国的粮食生产价格基本上围绕国家收购价格 进行波动, 粮食收购保护价格是在粮食生产成本上加入一定的利润, 因此物质费用成本上升 对粮食生产价格的影响很明显。

化肥成本和机械作业费用是粮食生产物质成本中的主要构成部分, 2014 年两者之和占物 质成本的 64\%, 物质成本中化肥所占比重最大。40 年间每亩化肥投资额增长到期初的 28 倍, 主要原因是化肥价格的大幅上涨。相比之下机械作业费的增长更加迅猛, 2014 年每亩地的机 械费用是 1975 每亩地机械费用的 138 倍, 在所有的物质费用中机械费用增长最快 [9]。除了 化肥成本和机械作业费之外, 物质成本中增长较快的是灌溉作业费用和农药费用, 二者分别 增长到原来的 21.4 倍和 18.3 倍, 而种子种苗费在 40 年间增长的幅度和粮食生产价格的增长 速度基本相同，其所占物质成本的比重变化不大基本上维持在 $12 \%$ 的水平。

从以上分析可以看出上游要素价格的上涨对粮食作物的生产成本影响很大, 而粮食作物生产 成本之所以没有同步增长相同的幅度主要是技术进步的因素, 技术进步减少了对这些要素的 依赖, 提高了农民对要素价格上涨的承受能力。

(2) 三种主要粮食作物对非传统物质要素的变化及影响

不仅传统的物质要素价格上涨会推动粮食作物的成本上涨, 人工成本和土地成本也大大推 动了粮食作物的成本。自 2003 年以来, 土地成本每年 13.08\%的增长速度远远超过了物质要 素 $7.60 \%$ 的整体增长速度。人工成本的增长速度明显低于物质成本和土地成本的增长速度, 从 2003 年的 $36.51 \%$ 递减到 2014 年的 $41.81 \%$ 。化肥投入、机械费用、灌溉成本、农药费用、 种子种苗分别属于物质成本的一部分; 物质成本与人工成本之和为生产成本; 生产成本与土 地成本之和为总成本。

\section{2 三种主要粮食作物消费价格的成本因素分析}

粮食生产价格是推动粮食消费价格增长的重要动力, 但不是唯一的动力。 生产价格在 25 年间仅仅增长到了原来的 3.35 倍, 略高于居民消费价格的增长, 只有粮食消 费价格增长的 45\%。假定粮食销售经济体每年的利润率基本相同, 那么必然有其他因素推动 粮食的最终消费价格发生变化, 否则很难解释消费价格的增长, 这些其他因素不属于粮食生 产领域，而是属于粮食流通领域，即流通成本是粮食消费价格提高的重要推手（表 1）。

表1 三种主要粮食作物平均每亩成本变动统计表(元)

\begin{tabular}{|l|c|c|c|c|c|c|c|c|}
\hline 统计对象 & 统计类别 & 1975 & 2003 & 2009 & 2014 & $\begin{array}{c}1975-2014 \\
\text { 平均增长速度 }\end{array}$ & $\begin{array}{c}2003-2014 \\
\text { 平均增长幅度 }\end{array}$ & $\begin{array}{c}2009-2014 \\
\text { 平均增长幅度 }\end{array}$ \\
\hline 化肥投入 & 每亩投入绝对额 & 4.7 & 57.93 & 117.55 & 132.42 & 8.94 & 7.81 & 2.41 \\
\hline 机械费用 & 每亩投入绝对额 & 1.02 & 24.09 & 72.6 & 124.92 & 13.12 & 16.14 & 11.47 \\
\hline 灌溉成本 & 每亩投入绝对额 & 0.91 & 14.72 & 19.45 & 25.62 & 8.94 & 5.17 & 5.67 \\
\hline 农药费用 & 每亩投入绝对额 & 1.13 & 9.22 & 20.66 & 27.56 & 8.53 & 10.47 & 5.93 \\
\hline 种子种苗 & 每亩投入绝对额 & 2.95 & 19.07 & 33.58 & 57.82 & 7.93 & 10.61 & 11.48 \\
\hline 物质成本 & 每亩投入绝对额 & 23.26 & 186.64 & 297.4 & 417.88 & 7.69 & 7.6 & 7.04 \\
\hline 人工成本 & 每亩投入绝对额 & 24.7 & 137.66 & 188.39 & 446.75 & 7.71 & 11.3 & 18.85 \\
\hline 生产成本 & 每亩投入绝对额 & 47.96 & 324.3 & 485.79 & 864.63 & 7.7 & 9.32 & 12.22 \\
\hline 土地成本 & 每亩投入绝对额 & & 52.73 & 114.62 & 203.94 & & 13.08 & 12.21 \\
\hline 总成本合计 & & 50.49 & 377.03 & 600.41 & 1068.57 & 8.14 & 9.93 & 12.22 \\
\hline
\end{tabular}




\section{3 生产成本的变化及其对农民收益能力的影响}

虽然粮食的生产价格在 40 年间发生了重大变化, 但是在粮食价格上涨最强烈的几年里粮 食生产价格的增长幅度却落后于粮食生产成本的增长幅度。图 2 是三种主要粮食作物每 50 公 斤主产品的平均纯收益率在 40 年间的走势图。根据走势图可以看到中国农民的收益能力在这 一期间大概经历了三个阶段: 1975-1985 年之间是收益能力大幅增长的阶段, 1985-1995 年之 间是纯收益率高位震荡的阶段; 1995 年之后的十几年是收益能力大幅下降并低位徘徊的阶段。 由于最近十多年粮食生产价格上涨不足以弥补生产成本上涨的损失, 生产价格的滞涨严重制 约了农民的投资收益能力, 从 1975 年到 2014 年每亩主要粮食作物的生产成本增长了 9.12 倍, 总成本增长了 18 倍（图 2）。

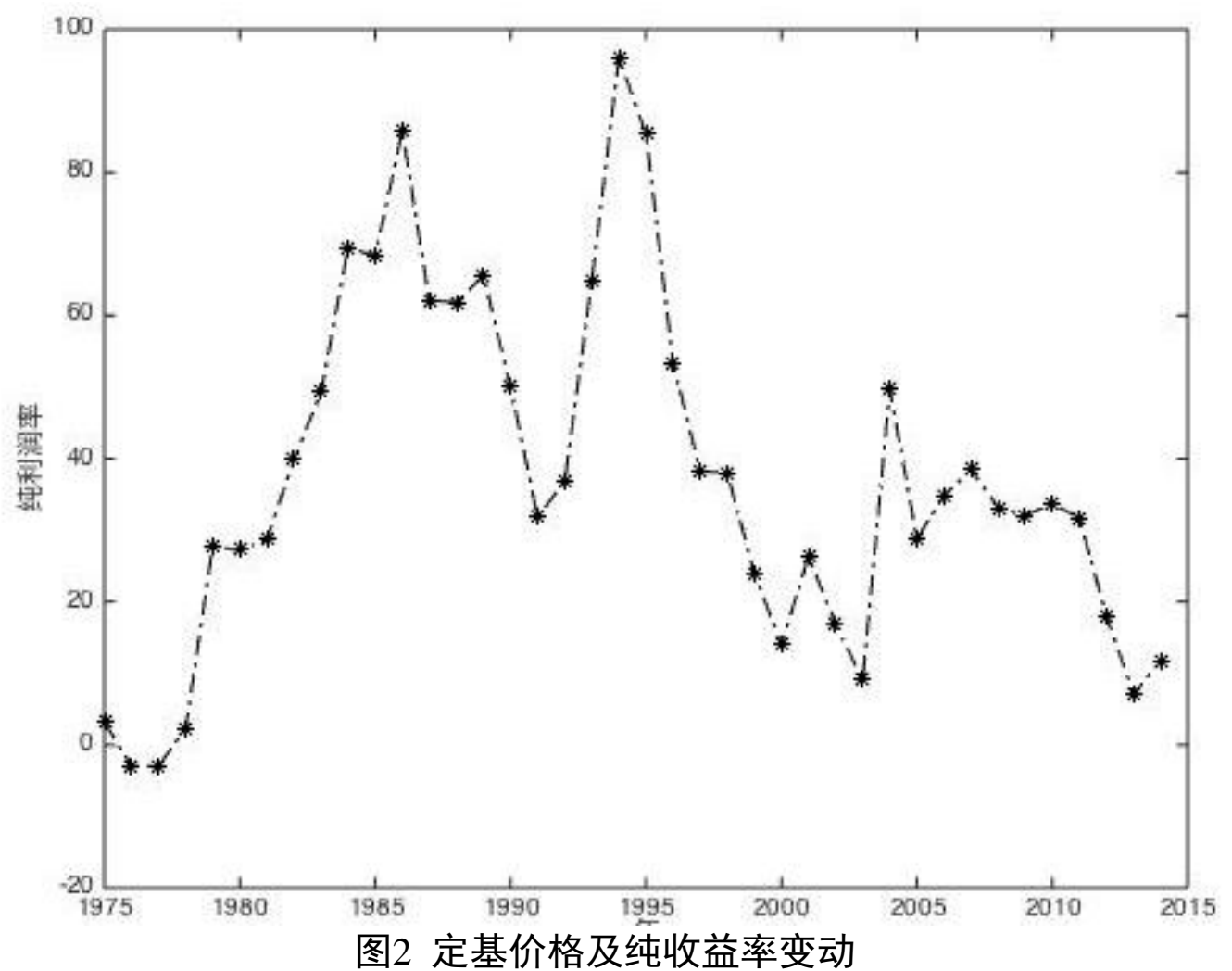

\section{4 粮食价格对居民消费 CPI 的影响分析}

人们往往认为粮食生产价格上涨推动 CPI 提高, 事实上粮食生产价格对 CPI 的影响并不明 显, 研究证明与 CPI 高度相关的是粮食消费价格 $[10,11]$ 。虽然成本上升导致了粮食生产价格 的增长速度略高于 $\mathrm{CPI}, 25$ 年间平均每年高出 $0.8 \%$, 这还不足以引起社会的广泛关注, 真正 让粮食价格成为热点的是粮食的消费价格。作为居民消费价格的一部分, 粮食消费价格从 1990 年以来的增长速度远远高于居民消费价格的整体增长速度。2 5 年间粮食消费价格增长到原来 的 6.37 倍, 而整体的居民消费价格仅仅增长到 1990 年的 2.80 倍, 粮食消费价格增长的速度 超过整体价格增速的两倍。全国居民消费价格 25 年间平均增速为 $4.21 \%$, 粮食生产价格的平 均增速为 $4.96 \%$, 而对应的粮食消费价格平均增速为 $7.69 \%$ （图 3）。 


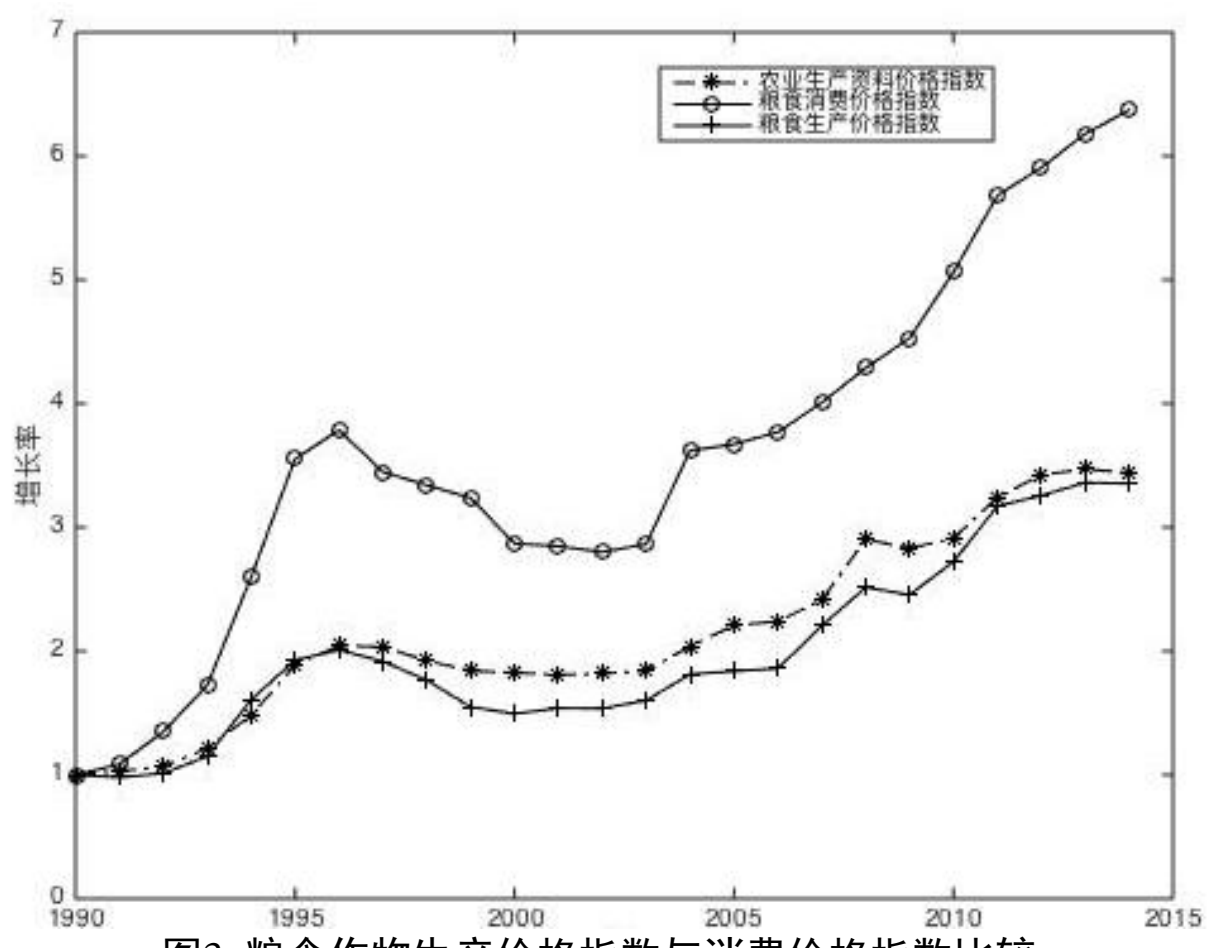

图3 粮食作物生产价格指数与消费价格指数比较

\section{4. 结论与分析}

4. 1 粮食消费价格成本推动粮食消费价格上涨（图 4)

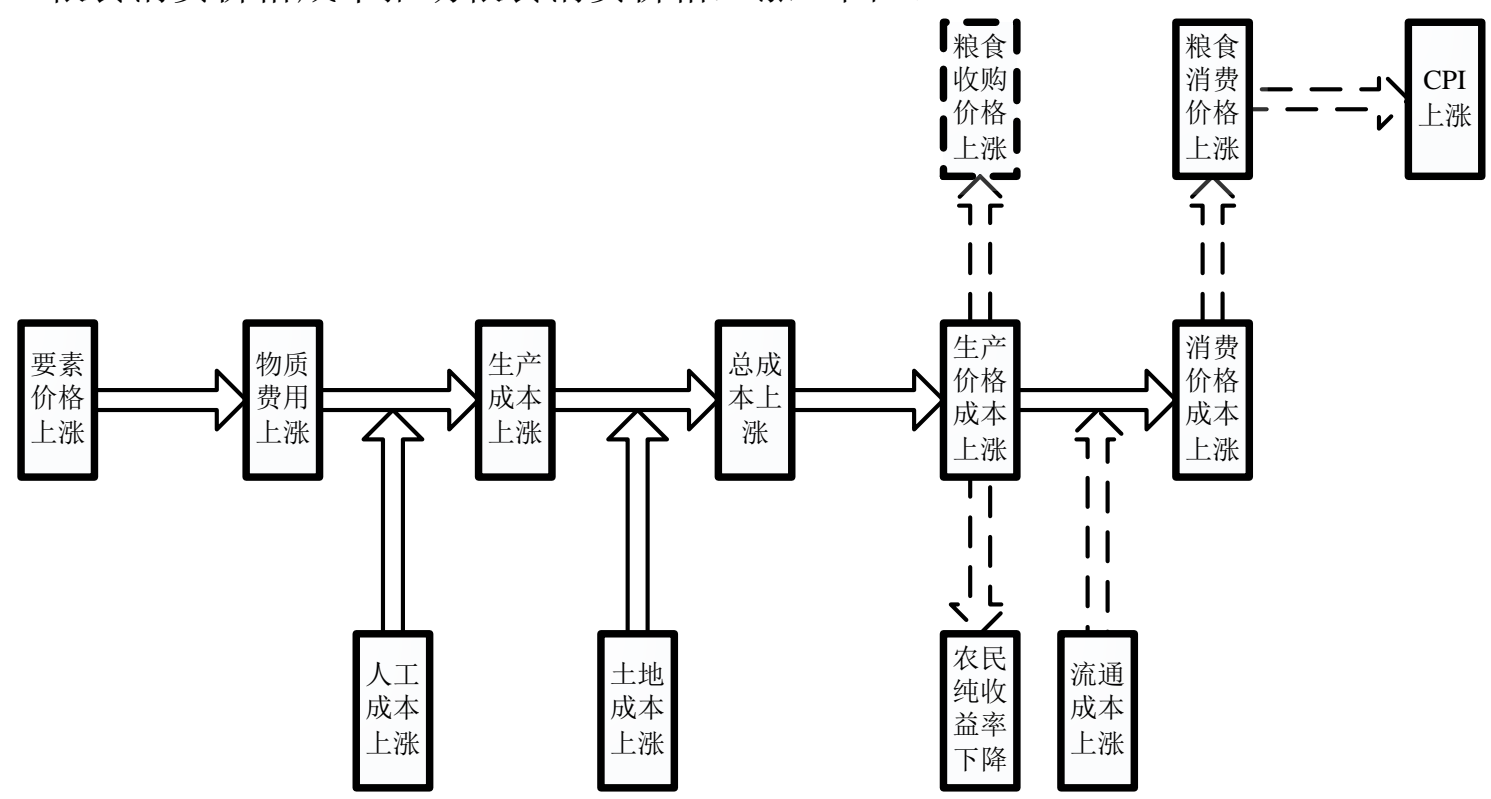

图4 粮食作物的不同成本和价格之间的传导机制

根据前面的分析可以得出图 4 的粮食作物的不同成本和价格之间的传导机制。因为粮食的 生产成本对粮食消费价格的影响体现在生产环节，粮食生产成本的全部影响体现在粮食生产 价格的变动上，粮食的生产成本与农业生产资料价格指数的变动的方向和程度基本一致（图 3）。而粮食的生产价格与粮食的消费价格之间存在很大的差异。生产成本不是推动粮食消费 价格大幅上涨的主要因素。

物流成本的特点表现在三个方面：一、物流效率底下。2005 年我国物流成本是发达国家 的一倍, 每年有总值 750 亿元人民币的食品在运输过程中腐坏, 食品企业的物流费用占到食 品成本的 70\%。而按照国际标准，食品物流成本最高不能超过食品总成本的 50\% [12]。二、流 
通费用上升。我国公路处处收费, 中国的城市化进程使郊区和城区的距离加大, 都增大了流 通成本 [13]。三、物质成本上升。国际国内油价的大幅上涨必然导致运输成本上升。

\section{2 农民并没有从粮食消费价格上涨中得到益处}

粮食生产价格的涨幅远远小于粮食消费价格的涨幅, 农民作为生产环节的受益人, 并没有 从粮食消费价格上涨中得到太多的益处, 相反农民承担并消化了农业生产要素价格带来的成 本压力 $[14,15]$ 。因此降低粮食的收购价格是不现实也是不公平的。三种主要粮食作物在 2014 年每亩净利润比 2003 年降低了很多, 每亩的纯收益率自 1994 年以来整体趋势是下跌的, 即 农民的获利能力越来越低。

\section{3 中国的粮食收购市场不是完全的竞争市场}

有人认为中国粮食市场 100\%的市场化了，粮食生产价格是市场竞争的结果而与政府无关。 但是，中国粮食市场即使市场化了也是寡头垄断的市场，极少存在不受政府干预的市场 [16]。 由于中国的粮食储备主体单一, 每年的粮食产量中很大一部分被中储粮收购, 政府很有效的 干预了粮食市场，只不过干预的时候行政化和市场化相结合了。

\section{4 工农业剪刀差的思想和影响仍然存在}

工农业剪刀差的思想仍然存在。首先体现在粮食收购市场上, 长期以来收购主体单一, 中 储粮处于垄断地位, 而中储粮的主要工作是执行政策性收粮, 其目的是为了保证国家的粮食 生产价格稳定，但是这种稳定往往是以牺牲农民的利益为代价的，不利于农业的长治久安。 由于中储粮执行的所谓粮食保护价格低于市场收购价格, 包括中粮油在内的其它收购企业往 往为了保证中储粮的收购而面临政策性的停购, 或者为了避免竞争而受到政府的整顿, 甚至 中粮储自己的商业性收购也被整顿，政府干预行为表现的很突出。

\section{5. 相关政策建议}

\section{1 中国的粮食收购市场应该进一步市场化}

由于粮食生产价格并非 CPI 的最终推动因素, 为了彻底改变农民投资收益能力下降的局 面，让农民成为粮食消费价格上涨的受益者，政府应采用改革的思维，进一步放开粮食收购 价格，放开粮食市场，形成自由竞争的农产品购销市场。

\section{2 改变工农业剪刀差的思维模式，体现真正的农工平等}

以农补工的工农业剪刀差的思维和政策一直存在, 经过 30 多年的改革开放, 中国已经进 入工业化中后期, 现在的中国已经到了以工补农的阶段了, 对农业的历史欠账必须要补回来 才是最符合中国的大局利益的, 因为农业受益的对象是中国最广大的农民群体, 关系的涉及 整个社会安稳的粮食安全问题。

\section{3 大力降低流通环节的成本}

中国降低粮食消费价格的当务之急是需要降低粮食流通环节的成本。一方面需要政府通过 建立现代物流平台, 打破交通运输领域的割据和垄断, 降低公路收费。另一方面增加国家粮 食储备的主体，减少粮食系统体制性的腐败和高成本。

\section{References}

[1] Li Guoxiang. Improve the agricultural support system to balance and stabilize theprices of agricultural products [J]. China economic \& trade herald, (2010) No. 06, p. 14-15.

[2] Tang Bulong. An empirical study on the relationship between logistic cost and price of agricultural products [J]. Journal of Huaiyin teachers college (Philosophy and social science edition), (2009) No. 5, p. 618-620.

[3] Hu Zhuohong, Shen Shijun. Research on the circulation cost of the price rising of agricultural products [J]. Price theory \& practice, (2010) No. 3, p. 27-29. 
[4] Hu Feng. A comparative study on the fluctuation of grain production and consumption price index in China (1990-2009) [J]. Liaoning Economy, (2011) No. 01, p. 47-49.

[5] Capehart, Tom, Richardson, Joe. Food Price Inflation: Causes and Impacts [J]. Congressional Research Service Reports, (2008) No. 4.

[6] Xia Zhicui. Analysis on the cost structure and price level of agricultural products in China [J], Price theory \& practice, (1995) No. 5, p. 38-40.

[7] Clifton B. Luttrell. Farm price supports at cost of production [J], Federal Reserve Bank of St Louis Review, (1977) No. 12, p. 2-7.

[8] Wang Shuyan. Analysis on factors of fluctuation and prediction on food prices in China [D]. Harbin: Northeast Agricultural University, China, 2013.

[9] Huang Jikun, Ma Hengyun. Comparison of major agricultural production costs and major international competitors [J]. Chinese rural economy, (2000) No. 5, p. 17-21.

[10]Li Guangsi, Zheng Yusheng. Food price regulation, system costs and social welfare: based on the analysis of two price policies [J]. Agricultural economy, Vol. 35 (2014) No. 08, p. 6-15.

[11]Su Zhifang, Wang Xiang, Chen Changnan. Study on the influence factors of low frequency fluctuation of grain price in China: based on panel VAR model [J]. Journal of agrotechnical economics, (2012) No. 10, p. 22-30.

[12] Yang Ju, Wang Xinkui. Effectiveness of government regulation of grain market in China: an empirical study based on wheat [J]. Economic research, (2008) No. 8, p. 42-49.

[13] Yan Wenjian, Li Hongyu, Li Xingmin. Food logistics and food safety [J]. Science and technology of food industry, (2006) No. 5, p. 24-26.

[14]Peng Chanjuan, Xu Xuerong. Analysis on the influencing factors of grain price fluctuation in China [J]. Price theory \& practice, (2015) No. 09, p. 67-69.

[15]Deng Dacai. Research on the effect of increasing grain price change: 1978-2004[J]. Economist, (2009) No. 02, p. 48-53.

[16]Zhang Ruijuan, Ren Xiaona. Research on pricing mechanism and volatility of grain products: a literature review and comments [J]. Journal of China agricultural university, Vol. 21 (2016) No. 01, p. 141-146. 